\title{
Pasos hacia la institucionalización de la evaluación del impacto en salud en España: la percepción de los profesionales de salud pública
}

\author{
Steps towards the institutionalization of health impact assessment \\ in Spain: perception of public health professionals
}

\author{
E. Sanz ${ }^{1,2}$, S. Esnaola ${ }^{1}$, E. Aldasoro' ${ }^{1}$ A. Bacigalupe ${ }^{1}$, J. Zuazagoitia ${ }^{3}$
}

\section{RESUMEN}

Fundamento. La actual reforma normativa de la salud pública en España ha previsto la inclusión de la Evaluación del Impacto en Salud (EIS). El objetivo de este estudio es describir la visión de los profesionales de salud pública y planificación sobre la utilidad y aplicabilidad de la EIS en su trabajo diario y con ello reflexionar sobre la mejor manera de institucionalizarla.

Metodología. Se han desarrollado 3 talleres de formación en EIS dirigidos a profesionales de las diferentes ramas de la salud pública. Se ofreció un cuestionario para cumplimentar por las personas asistentes.

Resultados. El 41\% de las personas asistentes contestaron al cuestionario. Entre quienes respondieron, el 92\% consideró que el modelo de los determinantes sociales de la salud resulta de gran utilidad en el ámbito de la salud pública. Las principales vías identificadas para introducir la EIS fueron: la obligatoriedad normativa (85\%), la integración en otras herramientas de evaluación ya existentes (65\%) y la sistematización de esta metodología (40\%). En relación a la aplicabilidad de la EIS, se propusieron estrategias específicas para su introducción en las diferentes ramas de la salud pública.

Conclusiones. La institucionalización exitosa de la EIS requerirá de la opinión y colaboración del personal técnico. Se constata la aprobación generalizada de esta herramienta para su utilización en la administración. Para garantizar la viabilidad de su implementación, será necesario sensibilizar a los profesionales sobre el modelo de los determinantes sociales de la salud, y recoger sus demandas y sugerencias.

Palabras clave. Evaluación del impacto en salud. Salud en todas las políticas. Determinantes sociales de la salud. Políticas públicas.

\begin{abstract}
Background. The current normative reform of public health policy in Spain envisages the inclusion of Health Impact Assessment (HIA). This study contributes the vision of public health and planning professionals on the usefulness and applicability of HIA in their daily work and thus reflects on how best to institutionalize it.
\end{abstract}

Methodology. Three training workshop were carried out with public health professionals. Participants were specialists in different branches: epidemiological surveillance, environmental health, food safety and health promotion. In this HIA workshop, a questionnaire was given to the attendees to be completed.

Results. Forty-one percent of the people attending answered the questionnaire; $92 \%$ of the staff considered that the model of social determinants of health is useful in the field of public health. The principal routes for introducing HIA were: mandatory rules (85\%), integration into other existing assessment tools (65\%) and the systematization of this methodology (40\%). Concerning the applicability of HIA, specific strategies are proposed for its introduction into the different branches of public health.

Conclusions. The successful institutionalization of HIA will require the opinion and collaboration of the technical personnel. A widespread approval of this tool for its utilization in the administration was verified. To guarantee the viability of its implementation, it will be necessary to raise the awareness of professionals about the model of the social determinants of health, as well as to attend to their demands and suggestions.

Key words. Health impact assessment. Health in all policies. Social determinants of health. Public policy.

\section{An. Sist. Sanit. Navar. 2012; 35 (3): $403-412$}

1. Servicio de Estudios e Investigación Sanitaria. Departamento de Sanidad y Consumo del Gobierno Vasco.

2. Departamento de Sociología. UPNA.

3. Dirección de Salud Pública. Departamento de Sanidad y Consumo del Gobierno Vasco.

Recepción: 18 de enero de 2012

Aceptación provisional: 28 de febrero de 2012

Aceptación definitiva: 16 de mayo de 2012

\section{Correspondencia:}

Elvira Sanz Tolosana

Edificio Departamental de los Magnolios

Departamento de Sociología

Universidad Pública de Navarra

31006 Pamplona

elvira.sanz@unavarra.es 


\section{INTRODUCCIÓN}

Abordar los nuevos retos de la salud pública no puede ser una tarea exclusiva del sector sanitario ya que la salud está principalmente determinada por factores externos al mismo ${ }^{1-5}$. La estrategia Salud en Todas las Políticas (SeTP) ${ }^{6}$ propone incluir de forma sistemática la salud en todas las políticas sectoriales, promoviendo la acción intersectorial y transversal en aras a mejorar la salud de la población a través del abordaje de los determinantes de la salud y de esta forma crear un sistema de salud rentable y sostenible. Una herramienta para su desarrollo es la evaluación del impacto en la salud (EIS). La EIS se define como "una combinación de procedimientos, métodos e instrumentos que permiten juzgar los posibles efectos de una intervención (política, un programa o proyecto) en la salud de una población, y la distribución de los potenciales efectos en la misma”, es decir, considerando el impacto de la intervención sobre las desigualdades sociales en la salud ${ }^{7-11}$. La EIS es una herramienta de carácter predictivo cuya aportación principal es que posibilita que las decisiones políticas estén basadas en pruebas para minimizar los efectos negativos sobre la salud, maximizar los positivos, y reducir el impacto en las desigualdades en salud de las intervenciones. Las experiencias europeas $^{12}$ y españolas ${ }^{13-19}$ muestran que la EIS es una herramienta muy flexible, de gran ayuda en la toma de decisiones saludables y justas, y que contribuye a la mejora de la salud de la población.

La reforma normativa y organizativa de la salud pública en España es una gran oportunidad para impulsar la introducción y desarrollo de la EIS ${ }^{20-21}$. La Ley General de Salud Pública (LGSP) del Ministerio de Sanidad $^{22}$ y la de Baleares ${ }^{23}$ ya la han incluido así como los anteproyectos de ley de Andalucía ${ }^{24}$ y del País Vasco ${ }^{25}$. El proceso a seguir para la implantación e institucionalización de la EIS en España es un debate abierto que necesita una mayor investigación y reflexión. En la mayor parte de los países, esta metodología se ha introducido a partir de documentos o programas específicos en los cuales los dirigentes políticos apoyan su desarrollo. No obstante, en ciertos ámbitos regionales de Bélgica, Alemania, Polonia, Eslovaquia así como en Québec, la EIS se ha institucionalizado al incorporarla en su Ley de Salud Pública ${ }^{26-28}$. La experiencia en la implantación de otras metodologías nos muestra que será necesario poner especial cuidado para evitar el rechazo o la burocratización que pudieran derivarse de la mera imposición de su aplicación. La aplicación de la EIS en las administraciones públicas exigirá la implicación decidida de los profesionales y los responsables políticos de sectores no sanitarios.

El proceso de institucionalización de la EIS en el País Vasco se encuentra en su fase inicial. Además de varias experiencias en el desarrollo de la EIS $^{13,17,19}$ y su inclusión en el anteproyecto normativo en el que se recogen los supuestos de aplicación previstos, se consideró relevante implicar a los profesionales. En ese contexto, se realizó un taller de formación sobre la EIS dirigido al personal técnico de salud pública y planificación sanitaria de la Comunidad Autónoma del País Vasco (CAPV). Este artículo tiene como objetivo presentar la percepción y opinión de los y las profesionales participantes sobre la utilidad y aplicabilidad de la EIS.

\section{METODOLOGÍA}

El taller denominado "Evaluación de impacto en salud: conceptos básicos y experiencias en la CAPV" se celebró en las tres capitales de la Comunidad Autónoma Vasca (San Sebastián, Vitoria y Bilbao), a lo largo del mes de junio de 2010. El taller tuvo dos objetivos: 1) presentar las bases conceptuales de la EIS, así como las experiencias desarrolladas en la CAPV, y enmarcarlas en la estrategia de "Salud en Todas las Políticas" y en la estrategia integrada de Prevención y Promoción de la Salud del Departamento de Sanidad y Consumo; y 2) recabar las opiniones del personal técnico sobre la EIS y su potencial aplicación en sus respectivas áreas de trabajo. La duración del taller fue de 10 horas en modalidad semipresencial y con una metodología activo- 
participativa. Tras la realización del taller, a modo de ejercicio práctico obligatorio para obtener la acreditación del curso, se les requirió a las personas asistentes que contestaran a un cuestionario (Tabla 1) validado y elaborado por el equipo del taller.

Tabla 1. Preguntas del cuestionario

1. Describe en un párrafo el modelo de los determinantes sociales de la salud. ¿Consideras necesario que este modelo se tenga en cuenta en el desarrollo de tu trabajo?

2. Vías para introducir la EIS. Nombra un par de áreas de trabajo en las cuales veas posibilidad de utilizar la herramienta EIS.

3. ¿Qué barreras observas para su implementación?

4. Resultados esperados. ¿Qué beneficios crees que se podrían obtener de la aplicación de la herramienta?

Los resultados del cuestionario fueron analizados conforme a la técnica de análisis de contenido temático (lista de frecuencias de repetición de categorías, e identificación y clasificación temática) y con el programa informático Atlas-ti. El número total de asistentes al taller fue de 80 personas. El criterio de selección utilizado fue el de trabajar en el ámbito de la salud pública. El número total de respuestas fue de $33 ; 16$ realizadas de forma individual y 17 realizadas en grupo. Las opiniones y recomendaciones contenidas en el texto se presentan en distintos bloques temáticos según los aspectos identificados más relevantes.

\section{RESULTADOS}

La tasa de respuesta global al cuestionario fue del $41 \%$. Todas las personas que respondieron cumplimentaron cada uno de los cuatro ítems. La mayoría fueron mujeres (74\%), de edades comprendidas entre los 37 y 63 años (media=48,6) y empleadas en el Departamento de Sanidad y Consumo del Gobierno vasco. La notación usada para las citas identifica a las perso- nas según el lugar de realización de los talleres (G1 hace referencia a Gipuzkoa, B1 a Bizkaia y V1 a Vitoria y los números a los distintos cuestionarios recogidos en cada uno de los lugares).

\section{El modelo de los determinantes sociales de la salud}

El 92\% del personal técnico consideró que el modelo de los determinantes sociales de la salud resulta de gran utilidad en el ámbito de la Salud Pública, ya que es un marco explicativo amplio e integral de los factores que intervienen en la salud. Además subrayaron su utilidad por la obligación de evaluar no sólo las intervenciones impulsadas desde el sector sanitario, sino también todas aquellas iniciativas que desarrolladas desde otros sectores pueden redundar en mayores o menores oportunidades de disfrutar de una vida en salud. Asimismo, argumentaron que el Plan de salud del País Vasco recoge la necesidad prioritaria de desarrollar políticas de salud encaminadas a reducir estas desigualdades, y para ello vieron necesario actuar desde este modelo. La adhesión a su posible implantación varía de intensidad: un participante la rechazó, 2 técnicos (6\%) consideraron que el hecho de no adoptarlo como marco de referencia es una "negligencia"; y $30(92 \%)$ lo valoraron como algo positivo y útil.

"Es evidente que no cabe otra respuesta que la de concluir que es totalmente necesario trabajar dentro de salud pública en la identificación de esas desigualdades y la utilización de la EIS como herramienta para tales fines" (G1).

\section{Vías para introducir la EIS}

La obligatoriedad normativa fue la principal vía identificada $(\mathrm{n}=28,85 \%)$ para la implantación y normalización de la EIS en la Administración y en el quehacer cotidiano de los profesionales. Proponían introducir esta herramienta por ley en todas las políticas, justificando que el cribado se encargará de determinar si es necesario o no realizar una EIS completa. Para argu- 
mentar su posición utilizaron como ejemplos la evaluación del impacto ambiental (EIA) y la de los riesgos laborales. En este sentido, la voluntariedad de su aplicación junto con el carácter no vinculante de sus resultados lo ven más como un problema. "Para ello requerimos de una normativa de apoyo. La labor técnica quedaría desvirtuada si no se dispone de elementos de presión para hacer cumplir las condiciones establecidas en el informe de conclusiones" (G5). Como elemento clave para su integración, destacaron la necesidad de una voluntad institucional, aspecto que hace referencia a la necesaria inversión económica (recursos humanos y materiales) y a la voluntad política para dar el impulso definitivo. Es lo que se ha denominado la vía de "apoyo desde arriba" (Fig. 1). Una segunda vía $(n=21,65 \%)$ es la de su integración en otras herramientas de evaluación existentes y operativas en la Administración, especialmente a través de la Evaluación de Impacto Ambiental (EIA), y/o su acoplamiento a las Autorizaciones Ambientales Integradas; a través de la Agenda 21 y de esta forma implementarla a nivel local o municipal, mientras que otros consideraron más factible su integración a través de los Planes de Ordenación del territorio. Y como tercera vía ( $n=13,40 \%)$, la estandarización y sistematización de esta metodología se vio como un paso necesario para la extensión de su aplicación. Consideraron que la ausencia de un procedimiento normalizado de aplicación de la EIS dificulta su introducción rutinaria. De hecho, la formación fue identificada como una pieza clave para su funcionamiento y su generalización (Fig. 1). Finalmente destacar la proposición de 2 técnicos (6\%) de hacer EIS a aquellos proyectos con demanda o polémica social (ej: la planta de coque de Petronor en Muskiz), y que un técnico de control y vigilancia no ve factible su aplicación en su área de trabajo.

En relación a la aplicabilidad de la EIS, las respuestas de las personas encuestadas aportaron información relevante en cuanto a la factibilidad de su implementación. Tomando en cuenta las diferentes áreas de trabajo en salud pública (vigi- lancia epidemiológica, sanidad alimentaria, sanidad ambiental y promoción de la salud), el personal técnico identificó vías explícitas para aplicar la EIS. En el área de vigilancia epidemiológica, el personal técnico demandó por un lado, incorporar nuevas variables a las encuestas y a los registros para generar indicadores fiables y útiles para la EIS y su evaluación témporo-espacial. Y por otro lado, se solicitaron canales de intercambio de información para el desarrollo de la EIS. Afirman que “...podríamos realizar alguna adaptación que nos permitiera mejorar la recogida, traducción, validación, análisis y difusión de la información para evitar que, al menos, ésta se convierta en un foco de posibles desigualdades en salud" (V5).

En el área de sanidad ambiental, el personal técnico valoró positivamente el valor añadido que supone la EIS. Sin embargo, la EIS es representada como un valor complementario a la EIA más que como herramienta independiente de evaluación.

"Pero, en los planes o programas ya sometidos a evaluación ambiental (principalmente los de ordenación territorial y urbanismo), se debiera aprovechar la evaluación ambiental para integrar la EIS. Si engranáramos las piezas para que ambos estudios de impacto pudieran integrarse, se produciría una sinergia de la que nos beneficiaríamos todos" (V3).

El personal del área de sanidad alimentaria apoyó la necesidad de evaluación que trae consigo la EIS como parte del trabajo en todas las áreas de trabajo relacionadas con los alimentos. Propusieron la integración de esta metodología evaluativa a todas las subáreas que la componen.

"Por otro lado, hay muchas otras áreas de trabajo relacionadas con los alimentos en la Dirección de Salud Pública en las que no es tan patente la importancia y la necesidad de una evaluación como parte del método de trabajo. Estas áreas se beneficiarían todavía más de la aplicación de un EIS al conseguirse de manera 'indirecta' una mejor planificación y evaluación” (V12). 


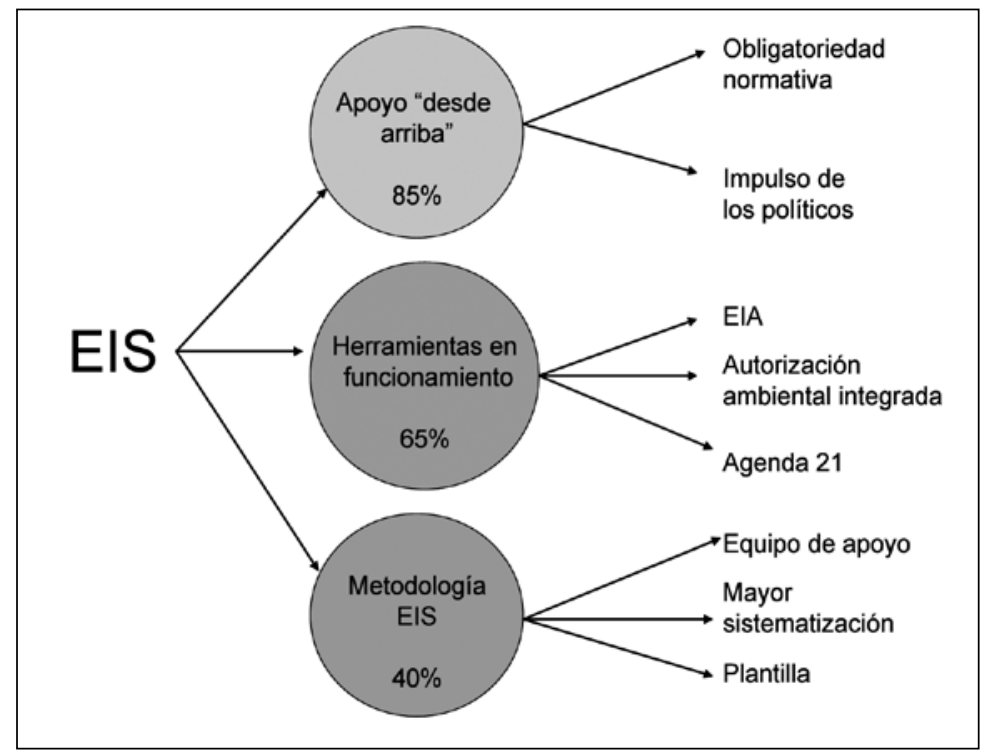

Figura 1. Vías de implantación de la EIS.

Y en el área de la promoción de la salud, la aceptación de esta herramienta y su rol de liderazgo en su desarrollo fue prácticamente unánime. "Que empiece la Unidad de promoción para luego una vez conseguida la obligatoriedad sea aplicada por todas las unidades" (B5).

\section{Barreras y dificultades}

Las dificultades identificadas por los profesionales de la salud para la implantación de la metodología EIS se podrían clasificar en dos grandes grupos en función de que éstas sean representadas como a) externas al trabajo del personal técnico, o b) como internas, en relación a la estructura jerárquica y cultura de la Administración Pública (Fig. 2).

\begin{tabular}{|c|c|}
\hline Externas (n, \%) & Internas (n, \%) \\
\hline $\begin{array}{l}\square \text { Falta de obligatoriedad normativa } \\
(28,85 \%) \\
\square \text { Falta de conocimientos del modelo de } \\
\text { los determinantes sociales de la salud } \\
(15,45 \%) \\
\square \text { Falta de decisión política, de liderazgo } \\
(10,30 \%) \\
\square \text { Falta de cooperación } \\
\text { interdepartamental }(5,15 \%) \\
\square \text { Coste }(3,9 \%)\end{array}$ & $\begin{array}{l}\square \text { Falta de formación/experiencias } \\
(9,27 \%) \\
\square \text { Falta de medios y de tiempo (9,27\%) } \\
\square \text { Resistencia/reticencia de los/las trabajadores al } \\
\text { cambio (5,15\%) } \\
\square \text { Estructura organizativa de la administración } \\
(4,12 \%) \\
\square \text { Falta de conocimiento/sensibilización de los } \\
\text { decisores políticos }(4,12 \%)\end{array}$ \\
\hline
\end{tabular}

Figura 2. Principales barreras para la implantación de la EIS 
a) La falta de sensibilización en el modelo de los determinantes sociales de la salud aparece como la principal causa que condiciona el desarrollo de la EIS. Una cuestión que no se limita de forma exclusiva a la clase política dirigente, sino que se extiende al resto de la población, e incluso al personal técnico. Sin embargo, la barrera externa más identificada fue la falta de obligatoriedad normativa. En relación a la clase política, el personal técnico critica su falta de liderazgo para impulsar esta herramienta en la administración vasca. Se senala como posible causa la incertidumbre generada por la EIS ante sus posibles resultados. "Posiblemente la principal barrera es la falta de interés de los promotores de las actividades a que se realice una EIS sobre lo que se plantean hacer. Esto es de aplicación tanto a las grandes infraestructuras como a una pequeña política que no se necesite de reflejo físico. Entre las posibles razones estarían:

1. Desconocimiento previo de las implicaciones de su realización, ya sean positivas como negativas.

2. Miedo a que implique un costo o cambiar el proyecto.

3. Miedo a la intervención de otros estamentos distintos al del promotor.

4. Miedo a que signifique un retraso en su realización

5. Por parte de los intervinientes, miedo a que sus aportaciones no se tengan en cuenta, en aras al bien mayor." (G3).

Por otro lado, la implementación de la EIS en la Administración pública se toparía con otro obstáculo importante. Dada la escasa experiencia y cultura de cooperación bajo la que subyace la lucha de competencias en este ámbito organizativo, la necesaria cooperación interdepartamental es representada como uno de los principales desafíos de esta herramienta. Para solucionarlo proponen establecer vías de colaboración, intercambio de información y el refuerzo de los elementos comunes. Y por último subrayar como barrera el coste de su implementación.

b) El personal técnico destacó las dificultades derivadas del propio carácter in- novador e incipiente de la EIS como son el desconocimiento de la metodología, la falta de experiencia y rodaje. A las que habría que sumar la falta de tiempo. Afirman que usar esta herramienta sobre un sistema que por definición tiene que mejorar la salud "les pone en la encrucijada".

También se afirma que la resistencia y/o reticencia de los trabajadores al cambio es un factor importantísimo a tener en cuenta. La falta de ilusión y motivación por afrontar nuevos retos fueron obstáculos identificados de forma significativa por los propios técnicos de la salud. Asimismo, se señaló a la estructura organizativa como factor que imposibilita su participación en el diseño y gestión de las actividades de promoción de la salud en general y que afectaría al desarrollo concreto de la EIS. Concretamente se hace referencia a la no inclusión dentro de sus funciones y tareas de aquellas relacionadas con la promoción de la salud. Finalmente, criticaron la falta de sensibilización y conocimiento sobre la EIS de los propios decisores políticos de sanidad.

\section{Beneficios esperados de la EIS}

Los beneficios que se esperaban tras la aplicación de la EIS coinciden mayoritariamente con los expuestos por la evidencia, es decir, maximizar las ganancias en salud (16,32\%), minimizar los efectos negativos que una intervención podría tener para la salud de la población $(10,30 \%)$, reducir las desigualdades en salud $(9,27 \%)$, y mejorar y facilitar la toma de decisiones $(9,27 \%)$. Opinaron que su aplicación será más beneficiosa tanto para las personas más desfavorecidas como para las áreas o zonas más deprimidas.

La inclusión de esta herramienta en el quehacer de salud pública requeriría la revisión de los actuales programas, una nueva organización del trabajo y de una nueva forma de trabajar: adoptar una visión integradora de la salud, la incorporación del valor salud en otros departamentos, colaboración intra e interdepartamental, participación ciudadana, y la optimización de los recursos. "Por otro lado el solo he- 
cho de trabajar para actuaciones o políticas no sanitarias incluyendo un punto de vista sanitario en ellas es de por sí un beneficio ya que hará que se tome en cuenta la salud como una responsabilidad social, y no sólo una responsabilidad individual o de políticas sanitarias concretas" (G10).

Asimismo, la incorporación de la participación ciudadana en la planificación de los programas y actuaciones mejoraría no sólo el conocimiento y el diagnóstico sino que también produciría un sentimiento de satisfacción tanto en la población, al sentirse implicada en la toma de decisiones y percibir una mayor protección sanitaria, como en el personal técnico. "Se revalorizaría la imagen de la Dirección de Salud Pública ante otros departamentos $e$ instituciones $y$ ante la población" (B9). "A nivel emocional individual de los trabajadores de las administraciones, una mayor satisfacción individual, de trabajar en conjunto y de una forma más coherente de lo que se está haciendo ahora" (B2). Y además, consideran que se haría un mejor uso de los recursos. "Se lograrian beneficios en la optimización de recursos dentro de nuestro ámbito de trabajo" (B9). "Además del beneficio en salud, el beneficio importante para nuestro trabajo es el instrumento en sí. Tener una herramienta común que nos ayude a valorar la influencia en la salud de proyectos o programas, ya que habitualmente actuamos por intuición y de acuerdo a nuestras experiencias, formación o área de trabajo, sin tener unos criterios unificados" (B3).

\section{DISCUSIÓN}

La aplicación de la EIS en las administraciones públicas exigirá la implicación de los profesionales por lo que se les ha de hacer partícipes conociendo y atendiendo sus demandas. En este sentido, este estudio constata que la utilidad del modelo de los determinantes sociales de la salud es mayoritariamente reconocida por el personal técnico. Las principales vías identificadas para introducir la EIS en la administración son la obligatoriedad normativa, el acoplamiento con herramientas ya instaladas y la sistematización de la herramienta. En rela- ción a la aplicabilidad de la EIS, se proponen estrategias específicas en las distintas ramas de la salud pública. Las principales dificultades previstas para la implantación de esta metodología se pueden clasificar en dos grupos: 1) externas (falta de obligatoriedad normativa, de sensibilización en el modelo de los determinantes sociales de la salud y de liderazgo); e 2) internas (falta de formación, de medios y de tiempo). Por otro lado, destacar que los beneficios esperados coinciden con la evidencia. En resumen, el estudio revela la aprobación generalizada de esta herramienta y la demanda de su aplicación en la administración por parte del personal de salud pública. La EIS emerge dentro de este colectivo como una herramienta efectiva para promover el bienestar, la salud y la equidad, y aplicable en todas las áreas de salud pública ${ }^{29}$.

El diseño exploratorio del estudio ha respondido al carácter novedoso e incipiente de esta metodología, y a la ausencia de trabajos de este tipo en el contexto estatal. Los resultados obtenidos en este trabajo no son representativos del colectivo del personal de salud pública del País Vasco, ya que la muestra no se obtuvo con criterios de representatividad, sino exploratorios. Sin embargo, los resultados permiten conocer cuáles son los principales factores (facilitadores y barreras) y discursos construidos en torno al desarrollo de la EIS. Unas representaciones sociales comunes a otras regiones por lo que sus resultados pueden ser extrapolables al resto de España. En este sentido, el estudio de las experiencias de implantación en otros países debe ser complementado con el análisis de la idiosincrasia española. Otra de las limitaciones importantes de este estudio hace referencia a las respuestas obtenidas del cuestionario. La mitad de ellas han sido respondidas de manera individual y la otra mitad grupalmente. Entendemos que los motivos para hacerlo de forma grupal responden a razones prácticas (falta de tiempo, sobrecarga de trabajo, etc.). El análisis de las respuestas no ha diferenciado si éstas eran individuales o grupales. Se ha considerado que la respuesta grupal es una respuesta consensuada. De esta forma, 
se ha podido perder parte de la argumentación sobre la EIS. A pesar de estas limitaciones, la fortaleza de este estudio reside precisamente en que recoge los aspectos claves con los cuales poder empezar a diseñar de forma satisfactoria las políticas para la institucionalización.

El estudio confirma la necesidad de sensibilización en el modelo de los determinantes sociales de la salud tanto del personal de sanidad como de la clase política como primer paso hacia la institucionalización de la EIS. Su práctica efectiva requiere superar el modelo biomédico de la salud y adoptar un modelo holístico que reconozca la relevancia de los determinantes sociales de la salud. La actual reforma normativa de la salud pública abre un camino para que la EIS adquiera la relevancia que le corresponde. Su inclusión en el ámbito normativo no sólo supone un avance importante para impulsar su desarrollo y su integración en la cultura organizativa. También responde a la demanda del personal técnico de contar con el respaldo legal necesario para afrontar su trabajo. Una oportunidad que debe valorar las dificultades de la implantación de otras metodologías para de esta forma explorar nuevas vías que eviten su posible banalización $^{30}$. La institucionalización de la EIS a través de medidas legislativas no garantiza el éxito de su implantación ${ }^{28}$. La viabilidad de su implementación en la administración también pasa por desarrollar tanto estrategias generales como estrategias adaptadas a las especificidades de los diversos ámbitos de la salud pública.La identificación de las dificultades internas responde al requerimiento del personal de una asignación adecuada de recursos (humanos, formativos, tiempo, etc.) que garantice su implementación. "Será necesario destinar recursos humanos suficientes pues de lo contrario se corre el peligro de convertir el procedimiento en un mero trámite a salvar" (V6).

La participación del personal técnico en el diseño e implementación de esta estrategia no sólo responde a una demanda intrínseca de la propia herramienta. Su participación junto a otros agentes sociales va a ser un catalizador para el desarrollo de la EIS. La amplia participación favorece la sensibilización de la opinión pública, impacta positivamente en el capital social y además ayuda a obtener un mejor diagnóstico y un mayor conocimiento sobre los determinantes sociales de la salud.

La necesaria colaboración interdepartamental presenta numerosos beneficios, pero también importantes obstáculos. La organización sectorial de la administración junto con la escasez de canales interdepartamentales de colaboración constituyen factores que dificultan notablemente la transversalidad necesaria para integrar la EIS. Como paso previo a esta cooperación, será necesario avanzar en la colaboración intradepartamental y evaluar sus resultados ("Estos programas los venimos ejecutando durante años pero nunca hemos hecho una evaluación de sus beneficios en salud" (B3).

La institucionalización de la EIS requerirá una nueva organización del trabajo y la adopción de una cultura más evaluativa, participativa e intersectorial. Un cambio en la cultura de la Administración que tendrá que contar con la participación ineludible de los profesionales. Y todo ello bajo una reformulación de la salud pública que adopte como eje vertebral la noción de Salud en Todas las Políticas.

\section{Agradecimientos}

El estudio se realizó gracias a la colaboración de la Dirección de Salud Pública del Gobierno Vasco y especialmente de su Directora Mercedes Estébanez Carrillo y de Beatriz Nuin; de las Subdirecciones Territoriales de Salud Pública de Álava, Bizkaia y Gipuzkoa; y por supuesto gracias a todas las personas asistentes a los talleres y que colaboraron respondiendo a los cuestionarios.

\section{BIBLIOGRAFÍA}

1. MaRmot M, WiLKInSON RG. Social determinants of health. 2nd ed. Oxford: Oxford University Press; 2006.

2. World Health Organization (WHO). Ottawa charter for health promotion. To move 
towards a new public health. An international conference sponsored by the World Health Organization, Health and Welfare Canada and the Canadian Public Health Association. Ottawa: 1986.

3. Mackenbach JP, Stirbu I, Roskam AJ, SchaAp MM, Menvielle G. Lein Salu M, et al. Socioeconomic inequalities in health in 22 European countries. N Engl J Med 2008; 358: 2468-2481.

4. Marmot M, Atкinson T, Bell J. Fair society, healthy lives. Strategic review of health inequalities in England post-2010. London: Secretary of State for Health, 2010.

5. Commission on Social Determinants of Health. Closing the gap in a generation. Health equity through action on the social determinants of health. Geneva: World Health Organization, 2008.

6. Stahl T, Wismar M, Ollila E, Lahtinen E, Leppok, eds. Health in all policies, prospects and potentials. Finland: Ministry of Social Affairs and Health. Health Department, 2006.

7. WHO European Centre for Health Policy. Health impact assessment. Main concepts and suggested approach. Gothenburg Consensus Paper. Copenhagen: WHO Regional Office for Europe; 1999.

8. Scott-Samuel S. Health impact assessment: theory into practice. J Epidemiol Community Health 1998; 52: 74-75.

9. Mindell J, Boltong A, Forde I. A review of health impact assessment frameworks. Public Health. 2008; 122: 1177-1187.

10. Bacigalupe A, Esnaola S, Calderón C, ZuazagorTiA J, Aldasoro E. La evaluación del impacto sobre la salud: una herramienta para incorporar la salud en las intervenciones no sanitarias. Gac Sanit 2009; 23: 62-66.

11. Esnaola S, Bacigalupe A, Sanz E, Aldasoro E, CalDERÓN C, ZuAZAGortia J et al. La evaluación del impacto en la salud: una vía para introducir la salud en todas las políticas. INFORME SESPAS 2010. Gac Sanit 2010; 24 (Supl.1): 109-113.

12. Wismar M, Blau J, Ernst K, Figueras J. The effectiveness of health impact assessment. Scope and limitations of supporting decision-making in Europe. Copenhagen: WHO regional Office for Europe; 2007.

13. Bacigalupe A, Esnaola S, Calderón C, Zuazagoitia $\mathrm{J}$, ALDASORO E. Health impact assessment of an urban regeneration project: opportunities and challenges in the context of a Southern European city. J Epidemiol Community Health 2010. 64: 950-955.

14. Díez E, Morteruel M, Artazcoz L, Borrell C, PaSARÍN M. Evaluación del impacto en salud de la ley de barrios en la Barceloneta. Gac Sanit. 2009; 23 (Especial congreso 1): 286-287.

15. VENEGAS J. HIA of an urban regeneration project in Alcalá de Guadaíra: a pilot experience in Andalusia. Comunicación presentada en 11th International Conference of HIA. 2011; Granada.

16. GonZÁlez R, Martín-Olmedo P, Guón Mt. Estudio prospectivo de la Evaluación de Impacto en Salud de la implantación del metro en la ciudad de Granada. Gac Sanit 2007; 21 (Supl. 2): 57.

17. Gómez F, Estibalez J. Evaluación del impacto en salud del aprovechamiento del espacio liberado por el soterramiento del ferrocarril en Vitoria-Gasteiz. Gac Sanit. 2009; 23 (Especial congreso 1): 9-10.

18. Boldo E, Aragonés N, Medina S, Pérez-Gómez B, Pollán M, Lope V et al. Evaluación de impacto en salud: una herramienta infrautilizada en salud pública. Ejemplo Apheis (Air Pollution and Health: a European Information System). Bol Epidemiol Sem 2005; 13: 97-108.

19. Aldasoro E, Sanz E, Bacigalupe A, Esnaola S, CAmbra $\mathrm{K}$ et al. Avanzando en la Evaluación del Impacto en la Salud: análisis de políticas públicas no sanitarias del Gobierno Vasco como paso previo a la fase de cribado. Gac Sanit 2012; 26: 83-90.

20. URBANos R. Recomendaciones para una futura Ley de Salud Pública en España. Gac Sanit 2010; 24: 89-94.

21. URbanos R. La salud en todas las políticas. Tiempo de crisis, ¿tiempo de oportunidades? Informe SESPAS 2010. Gac Sanit 2010; 24 (Supl. 1): 7-11.

22. Ley $33 / 2011$, de 4 de octubre, General de Salud Pública. Boletín Oficial del Estado, $\mathrm{n}^{\mathrm{O}} 240$ (05-10-2011).

23. Ley 16/2010, de 28 de diciembre, Salud pública de las Illes Balears. Boletín Oficial del Estado, $\mathrm{n}^{\mathrm{o}} 30$ (04-02-2011).

24. Junta de Andalucía. Anteproyecto de ley de salud pública. http://www.juntadeandalucia.es/ salud/anteproyectoleysaludpublicanavegable/

25. Gobierno Vasco. Anteproyecto de ley de salud pública. http://www.openideiak.com/pg/ groups/32458/elaboracion_del_anteproyecto_de_ley_de_salud_publica_de_la_comunidad_autonoma_del_pais_vasco/

26. WISMAR M. Implementing and institutionalizing HIA in Europe. En: Stahl T, Wismar M, Ollila E et al, eds. Health in all policies, prospects and potentials. Helsinki: Finnish Ministry of Social Affairs and Health; 2006. 
27. Wright J, PARry J, Scully E. Institutionalizing policy-level health impact assessment in Europe: is coupling health impact assessment with strategic environmental assessment the next step forward? Bull World Health Organ 2005; 83: 472-477.

28. Boldo E, St-Pierre L, Mendell A, Benoit F. La institucionalización de la evaluación del impacto en la salud en Quebec: ¿un modelo factible en España? Gac Sanit 2011; 25: 535-541.
29. Milner SJ, Bailey C, Deans J, Pettigrew D. Integrated impact assessment in the UK-use, efficacy and future development. Environ Impact Assess Rev. 2005; 25: 47-61.

30. SAnZ E. Perception of public health and health planning professionals about utilities and applicability of HIA. Comunicación presentada en 11th International Conference of HIA. 2011; Granada. 